\title{
Stellar feedback from a massive Super Star Cluster in the Antennae merger
}

\author{
Cinthya N. Herrera ${ }^{1} \dagger$ and Francois Boulanger ${ }^{2}$ \\ ${ }^{1}$ National Astronomical Observatory of Japan, \\ 2-21-1 Osawa, Mitaka, Japan \\ email: cinthya.herrera@nao.ac.jp, herrera@iram.fr (present address) \\ ${ }^{2}$ Institut d'Astrophyisque Spatiale \\ Université Paris Sud, Batiment 121, 91405 Orsay, France \\ email: francois.boulanger@ias.u-psud.fr
}

\begin{abstract}
Stellar feedback from massive stars can unbind and disperse large amount of molecular gas, affecting the star formation efficiency. Based on ALMA and VLT observations in the Antennae galaxies we study a massive $\left(\sim 10^{7} \mathrm{M}_{\odot}\right)$ and young $(\sim 3 \mathrm{Myr})$ SSC, B1, associated with compact molecular and ionized emission, which suggests that it is embedded in its parent cloud. However, we found contradictories and puzzling results on the structure and dynamics of the matter around the cluster, indicating that SSC B1 is not embedded in its parent cloud after all. We propose that radiation pressure was highly enhanced at the early stages of the SSC formation, disrupting the parent cloud in $<3$ Myr. We show evidences of outflowing gas from the parent cloud in the more extended CO gas. Higher angular resolution observations are needed to validate this interpretation and to understand the origin and fate of the component seen to be associated with SSC B1.
\end{abstract}

Keywords. ISM, galaxies: star clusters, interactions

\section{Introduction}

Star formation is an inefficient process. Observations of giant molecular clouds (GMCs) in the Milky Way suggest that the highest star formation efficiency (SFE) occurs in massive $\left(>10^{4} \mathrm{M}_{\odot}\right)$ GMCs reaching only $20 \%$ (Murray 2011). Many studies have considered stellar feedback as a main energy source to maintain turbulence as star clusters are formed and thus preventing star formation (e.g., Tan et al. 2006, Krumholz et al. 2006). Several stellar feedback mechanisms have been proposed and analytically quantified (e.g., photoionization and expansion of HII regions, stellar winds, SN explosions, radiation pressure). Eventually, the energy and momentum injected by the newly formed stars will unbind and disperse the matter around them. How quickly this occurs is key to the SFE.

Massive stellar feedback is important to galaxy evolution and star formation history in the universe. Observations of starburst galaxies suggest that $P_{\mathrm{rad}}$ is the main driving mechanism of galactic molecular outflows (e.g., Geach et al. 2014), dispersing and disrupting large amounts of molecular gas thus affecting the total SFE. At smaller scales, $P_{\text {rad }}$ is found to dominate within the shells close to the central SSC in the 30 Dor star forming region (Lopez et al. 2011). Numerical simulations also indicate that $P_{\text {rad }}$ would be the dominant stellar feedback mechanism close to massive star clusters where the escape velocity is larger than the sound speed of the HII gas (Krumholz \& Matzner 2009; Murray et al. 2010). However, it is still unclear how this occurs in GMC scales since observational support is insufficient.

$\dagger$ Present address: IRAM, 300 Rue de la Piscine, 38406 Saint Martin d'Hères, France. 


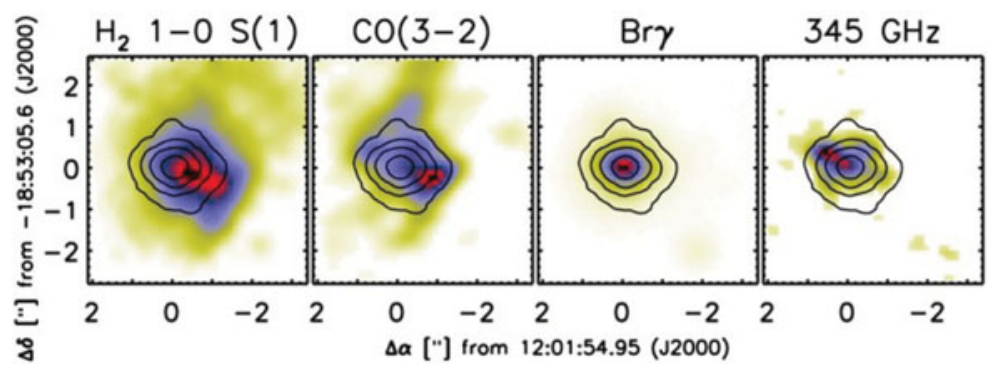

Figure 1. Intensity maps for SGMC $4 / 5$. From the left to right, $K$-band continuum emission contours overlaid on $\mathrm{H}_{2} 1-0 \mathrm{~S}(1), \mathrm{CO}(3-2), \mathrm{Br} \gamma$ and continuum emission at $345 \mathrm{GHz}$. The peak emission of the contours marks the position of SSC B1.

Super star clusters (SSCs) are one of the most extreme forms of star formation, being compact (few parsecs size) and massive $\left(>10^{5} \mathrm{M}_{\odot}\right)$. The are recurrently found in galaxy interactions, common phenomena in the Universe. SSCs must be ubiquitous in the Universe. SSCs in nearby mergers are ideal sites to study massive stellar feedback. The Antennae galaxy (NGC 4038/39) is a nearby (22 Mpc, Schweizer et al. 2008) merger between two gas-rich spiral galaxies that hosts a large number of SSCs (Whitmore et al. 1995). In the region where the two disks interact, the overlap region, large amount of molecular gas is observed to be fragmented into Super-Giant Molecular Complexes (SGMCs, Wilson et al. 2000), likely the birth places of SSCs. Interferometric observations show that SGMCs are clumpy and present different velocity components (Herrera et al. 2012). In this study we focus in the Antennae overlap region, where the most massive $\left(\geqslant 10^{5} \mathrm{M}_{\odot}\right)$ and youngest (<10 Myr) SSCs are located (Mengel et al. 2005).

\section{Observations}

We use two datasets on the Antennae overlap region. First, observations obtained with the SINFONI/VLT imager spectrometer facility, in the near-IR $K$-band which includes $\mathrm{Br} \gamma$ and several $\mathrm{H}_{2}$ rovibrational lines. Second, observations done with the ALMA interferometer in the Cycle 0 early science process (PI: B. Whitmore). These observations are done in Band 7 (345 GHz), which includes the $\mathrm{CO}(3-2)$ line and the dust continuum emission. Detailed information of these datasets can be found in Herrera et al. (2012) and Whitmore et al. (2014) for the VLT and ALMA observations, respectively.

\section{A unique SSC in the Antennae overlap region}

We search in our SINFONI field-of-views for SSCs still associated with compact molecular $\left(\mathrm{H}_{2}\right)$ and ionized $(\mathrm{Br} \gamma)$ gas emission. In SGMC $4 / 5$ we found a unique SSC, B1, which is also the most massive $\left(\sim 10^{7} \mathrm{M}_{\odot}\right)$ SSC in the overlap region. It is a bright target to study how massive SSCs disperse their parent molecular clouds.

Fig. 1 shows the distribution of the $\mathrm{H}_{2}, \mathrm{CO}, \mathrm{Br} \gamma$ and continuum emission. While the molecular gas extends across the SGMC, the ionized gas and $K$-band continuum are symmetrically distributed around the cluster. Fig. 2 presents the $\mathrm{CO}(3-2)$ channel maps where we can distinguish two spatially and spectrally separated components. The low velocity emission is mainly associated with SSC B1, while the higher velocity component seems to surround the cluster in a bubble-like shape structure. We hypothesize that the low velocity component traces the parent molecular cloud of SSC B1.

We estimate the mass of the GMC from the continuum emission at $345 \mathrm{GHz}$. We approximate the spectral dependence on the dust emission with a gray body and assume 


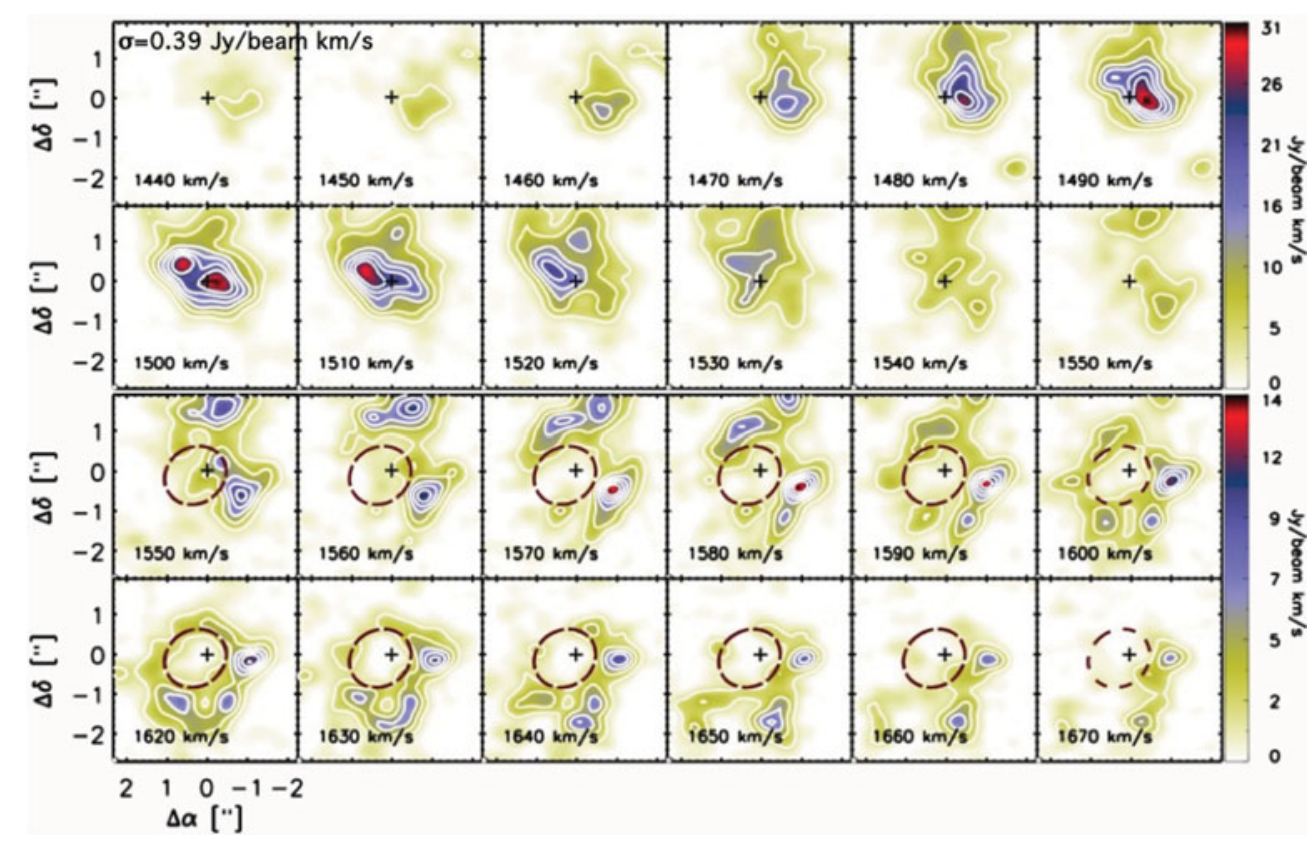

Figure 2. CO(3-2) channel maps of SGMC 4/5. The position of SSC B1 is marked with a cross. Top panel: channel maps from $1440 \mathrm{~km} \mathrm{~s}^{-1}$ to $1550 \mathrm{~km} \mathrm{~s}^{-1}$ by $10 \mathrm{~km} \mathrm{~s}^{-1}$. Contours start at $10 \sigma$ with a spacing of $10 \sigma$. Bottom panel: CO channel maps from $1550 \mathrm{~km} \mathrm{~s}^{-1}$ to $1670 \mathrm{~km} \mathrm{~s}^{-1}$ by $10 \mathrm{~km} \mathrm{~s}^{-1}$. Contours start at $5 \sigma$ with a spacing of $5 \sigma$. Offset position are the same as in Fig. 1.

that the dust properties in the Antennae are the same as those in the Galaxy. For a typical dust temperature of $20 \mathrm{~K}$, we derive a GMC mass of $4.6 \times 10^{7} \mathrm{M}_{\odot}$. We also estimate the molecular gas mass from the observed $\mathrm{CO}$ emission. Assuming the canonical $X_{\mathrm{CO}}$ conversion factor (Bloemen et al. 1986), we estimate the molecular mass to be $2.4 \times 10^{8}$ $\mathrm{M}_{\odot}, 5$ times larger than the value estimated from the continuum emission. This excess can be accounted for by a difference on the $X_{\mathrm{CO}}$ factor. Zhu et al. (2003) found a $X_{\mathrm{CO}}$ factor of at least 5 times smaller than the canonical value for the entire overlap region (at $1.5 \mathrm{kpc}$ scale).

\section{Physical structure of the matter surrounding SSC B1}

We describe the physical structure of the molecular gas surrounding SSC B1 which we associate with the low velocity component of the $\mathrm{CO}$ emission.

Gas pressure in the molecular gas. Radiation pressure is the pressure exerted by the stellar radiation over the molecular gas that surrounds the central star cluster. We quantify $P_{\text {rad }}$ in the molecular gas surrounding SSC B1 as $P_{\text {rad }}=\frac{L_{\mathrm{cl}}}{4 \pi \mathrm{R}_{\text {in }}^{2} \mathrm{c}}\left(1+\tau_{\mathrm{rad}}\right)$ (e.g., Murray et al. 2010), where $L_{\mathrm{cl}}$ is the luminosity of SSC B1, $\mathrm{R}_{\text {in }}$ is the HII radius and $\tau_{\text {rad }}$ accounts for the trapping of the IR photons within the cloud. We compute $\mathrm{P}_{\mathrm{rad}} / \mathrm{k}_{\mathrm{B}}=1.1 \times 10^{8}$ $\left(1+\tau_{\mathrm{rad}}\right) \mathrm{K} \mathrm{cm}^{-3}$.

We model the HII region surrounding the SSC as an ionized nebula with dust grains. For different values of $\mathrm{R}_{\text {in }}$, we solve the radiative transfer equation and measure the outward radiation field $(\chi)$ and $P_{\text {rad }}$ exerted at the surface of the photodissociation region (PDR). We compare our grid with the outputs of the PDR Meudon code (Le Bourlot et al. 2012), which predicts molecular line intensities from PDRs. We find that PDR 
models with $\chi=10^{3}-10^{4}$ times the mean radiation field in the solar neighborhood and a gas pressure of about $10^{8} \mathrm{~K} \mathrm{~cm}^{-3}$, are needed to account for the observed $\mathrm{R}_{\mathrm{S}(1)} \equiv \mathrm{H}_{2}$ 1-0/2-1 S(1) line emission. The $\chi$ value is consistent with that estimated by Gilbert et al. (2000) from the comparison of PDR models and several near-IR $\mathrm{H}_{2}$ line intensities from single-slit spectroscopy. The estimated gas pressure of $10^{8} \mathrm{~K} \mathrm{~cm}^{-3}$ agrees with the measured molecular gas pressure, supporting low values of $\tau_{\text {rad }}$. There is no significant trapping of the IR photons within the cavity of the molecular gas surrounding SSC B1.

Gas pressure in the hot plasma. SSC B1 was detected as a compact X-ray source by Chandra (Zezas et al. 2006). From its X-ray luminosity $\left(\mathrm{L}_{\mathrm{X}}=1.9 \times 10^{38} \mathrm{erg} \mathrm{s}^{-1}\right)$ we find an electron density of $0.6 \mathrm{~cm}^{-3}$. For a temperature of $\mathrm{T}=10^{7} \mathrm{~K}$, the pressure of the hot plasma is $P_{\text {hot, } \mathrm{x}} / \mathrm{k}_{B}=1.9 \mathrm{n}_{\mathrm{e}} \mathrm{T}=1.2 \times 10^{7} \mathrm{~K} \mathrm{~cm}^{-3}$.

The hot gas pressure is one order of magnitude weaker than the pressure of the molecular gas. This difference indicates that there is a leak of hot gas within the cavity. The parent molecular cloud is not a closed, homogeneous shell around the cluster, but it is already broken. This is consistent with our finding that $\tau_{\text {rad }}$ is negligible. This result also implies that the radius estimated for the molecular cloud is not an unique value. The molecular gas surrounding the cluster is clumpy, with molecular clumps located at different distances from the cluster.

\section{Outflowing gas}

The radiation pressure is not enough to compensate the gravitational attraction exerted by SSC B1 and the mass of the cloud itself. Today, $P_{\text {rad }}$ is weaker than the gravitational force and cannot push away the molecular gas associated with SSC B1.

We speculate that at least a significant part of the natal cloud has already been blown away and that the high velocity component is tracing outflowing molecular gas. This gas would have been earlier accelerated by $P_{\text {rad }}$ when it was much higher than today. We estimate the momentum of the outflowing gas from the mass in the CO high velocity component and the gas expansion velocity $v_{\exp }$ estimated from the line width of this component. To test the hypothesis that this gas was accelerated by $P_{\text {rad }}$ over a time scale of $t_{\text {feed }}$, we write $L_{\mathrm{cl}} / c\left(1+\tau_{\text {rad }}\right) t_{\text {feed }}=M_{\text {high }} v_{\text {exp }}$, where $t_{\text {feed }}=R_{\text {high }} / v_{\text {exp }}=2 \mathrm{Myr}$, with $R_{\text {high }} \simeq 100$ pc the mean size of the bubble-like shape structure observed in this component. We found a high value of $\tau_{\mathrm{rad}}$, possible when the parent cloud was not yet disrupted. (In the numerical models by Murray et al. (2010), $\tau_{\text {rad }}$ reaches values of several tens of units at the very beginning of the disruption of the matter). If we correct $M_{\text {high }}$ by the $X_{\mathrm{CO}}$ factor estimated by Zhu et al. (2003), $\tau_{\text {rad }}$ will be $\sim 2$. We estimate the outflow rate of the parent molecular cloud to be $18 \mathrm{M}_{\odot} \mathrm{yr}^{-1}$ (using the corrected $X_{\mathrm{CO}}$ factor), assuming a SFE for SSC B1 of $5-10 \%$.

The high velocity component is also observed in the $\mathrm{H}_{2} 1-0 \mathrm{~S}(1)$ emission. Fig. 3 shows the spectra for the $\mathrm{v}=1-0$ and $2-1 \mathrm{~S}(1) \mathrm{H}_{2}$ line emission. The $\mathrm{v}=2-1$ line can be fitted with a single Gaussian curve while $\mathrm{v}=1-0$ requires two components. The right panel shows in solid contours the high velocity component of $\mathrm{H}_{2}$ overlaid to the $\mathrm{CO}$ high velocity component. The spatial extent of both high velocity components are similar.

The $R_{S(1)}$ ratio is commonly used to disentangle between $\mathrm{H}_{2}$ gas heated by UV radiation or collisions (e.g., Herrera et al. 2011). We find $R_{S(1)}=0.36$ and $<0.18$ for the low and high $\mathrm{H}_{2}$ velocity components, respectively. The former ratio can be accounted for by UV heating of the gas in PDRs. The latter can be observed towards PDRs with high radiation fields $\left(>10^{4}\right)$ and gas pressure $\left(>10^{8} \mathrm{~K} \mathrm{~cm}^{-3}\right)$. However, since this component is extended and there is no evidence for extended massive stellar population, we favor 

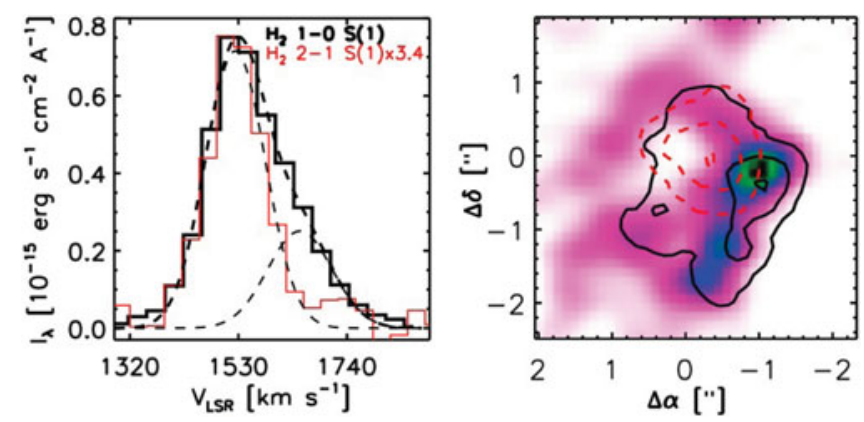

Figure 3. $\mathrm{H}_{2} 2-1 / 1-0 \mathrm{~S}(1)$ ratio in the SGMC 4/5. Left panel: spectra for $\mathrm{v}=1-0 \mathrm{~S}(1)(\mathrm{v}=2-1)$ $\mathrm{H}_{2}$ in thick line (thin line, scaled by a factor 3.4). The dashed lines are the gaussian fits for the spectra. Right panel: color image shows integrated intensity of the $\mathrm{CO}(3-2)$ high velocity component. Dashed and solid contours are the $\mathrm{H}_{2}$ emission integrated in $1300-1512 \mathrm{~km} \mathrm{~s}^{-1}$ and $1580-1760 \mathrm{~km} \mathrm{~s}^{-1}$, respectively. Offset position are the same as in Fig. 2.

shocks as main gas heating mechanism. Values in the range $0.1-0.2$ are reproduced in J- and C-shock models (Kristensen 2007) for gas with densities in the range $10^{4}-10^{6}$ $\mathrm{cm}^{-3}$ and shock velocities from 15 to $50 \mathrm{~km} \mathrm{~s}^{-1}$. This supports the idea that the high velocity component is tracing outflowing gas. Evidence of high velocity ionized gas can be observed in the $\mathrm{Br} \gamma$ line emission. At the velocities of the low velocity component $(<$ $1600 \mathrm{~km} \mathrm{~s}^{-1}$ ), the $\mathrm{Br} \gamma$ emission is symmetrical. An excess of ionized gas emission, where the $\mathrm{CO}(3-2)$ has a low column density, is observed at velocities of $1600-1700 \mathrm{~km} \mathrm{~s}^{-1}$, that we interpret as an evidence of escaping ionised gas.

\section{Discussion}

In our interpretation, today is too late to witness the direct disruption of the natal cloud of SSC B1. For a source of similar characteristics, Murray et al. (2010) predicted that the disruption occurs in $\lesssim 1 \mathrm{Myr}$. We argue that the outflowing gas from the disruption of the parent cloud is traced by the high velocity component. The bottom panel of Fig. 2 shows the channel maps of this component, which surrounds SSC B1 in a bubble-like shape structure with an inner radius of about 100 pc. Similar gas dynamics have been observed in the starburst galaxies M82 and NGC 253 (Weiss et al. 1999; Sakamoto et al. 2006), which have been interpreted as expanding super-bubbles.

Uncertainties on different parameters do not significantly impact our interpretation. First, there is an uncertainty on the $X_{\mathrm{CO}}$ factor, which implies an uncertainty on the molecular mass obtained from the CO luminosity for both velocity components. Second, there is an uncertainty on the efficiency of the formation of SSC B1. Even though low SFE (5\%-10\%) are common in Galactic molecular clouds, larger values have also been reported towards massive star forming regions. We have been conservatives by applying the commonly used values for both factors. By doing this, the results on the high velocity component are coherent indicating that most of this gas would be outflowing gas from the parent molecular cloud disruption. The non-detection of red-shifted gas towards the line of sight of the cluster, as typically observed in expanding shells, can be explained by the presence of the low velocity component. This component is very dense and it may be hiding the red-shifted outflowing gas. The origin and fate of the gas which is the closest to SSC B1, the low velocity component, is not clear since we cannot resolve structures smaller than $50 \mathrm{pc}$. In any case, the results on this component are somehow contradictories and thus difficult to interpret. Its virial parameter indicate that it is 
self-bound. However, the cluster escape velocity (at the distance of the internal cavity which is determined by the $\operatorname{Br} \gamma$ size) is $49 \mathrm{~km} \mathrm{~s}^{-1}$ while the observed CO line width is $59 \mathrm{~km} \mathrm{~s}^{-1}$, yielding an expansion velocity of at most $30 \mathrm{~km} \mathrm{~s}^{-1}$. Another source of confusion is given by a star forming region likely happening very close to SSC B1. Brogan et al. (2010) detected a water maser where the dust continuum (Fig. 1, peak emission at the position $[0.5,0.4]), \mathrm{HCO}+(4-3)$ line, and mid-IR continuum emission (Snijders et al. 2006) peak. The velocities of the $\mathrm{H}_{2} \mathrm{O}$ maser and the $\mathrm{HCO}+(4-3)$ line coincide. The no detection of $\mathrm{Br} \gamma, K$-band continuum nor $\mathrm{H}_{2}$ emission suggest that this source is an extremely young star forming region.

If our interpretation is not correct, we do not really understand how the cluster will get rid of its surrounding matter to match the observed values of SFE. This component is still very massive, comparable with the mass of the cluster, and even more it is self-bound. Further observations are needed to quantify and test our proposed scenario. Specifically, higher resolution observations will reveal the spatial distribution of the gas very close to the cluster, i.e. the gas observed in the low velocity component.

\section{References}

Bloemen, J. B. G., Strong, A. W., Blitz, L., Cohen, R. S., \& Dame, T. M., et al. 1986, A\&ऽA, 154,25

Brogan, C., Johnson, K., \& Darling, J. 2010, ApJ, 716, L51

Geach, J. E., Hickox, R. C., Diamond-Stanic, A. M., Krips, M., Rudnick, G. H., Tremonti, C. A., Sell, P. H., Coil, A. L., \& Moustakas, J. 2014, Nature, 516, 68

Gilbert, A. M., Graham, J. R., McLean, I. S., Becklin, E. E., Figer, D. F., Larkin, J. E., Levenson, N. A., Teplitz, H. I., \& Wilcox, M. K. 2000, ApJ, 533, L57

Herrera, C. N., Boulanger, F., \& Nesvadba, N. P. H. 2011, A\& A, 534, A138

Herrera, C. N., Boulanger, F., Nesvadba, N. P. H., \& Falgarone, E. 2012, A\&A, 538, L9

Kristensen, L. E. 2007, PhD thesis, LERMA, Observatoire de Paris-Meudon

Krumholz, M. R. \& Matzner, C. D. 2009, ApJ, 703, 1352

Krumholz, M. R., Matzner, C. D., \& McKee, C. F. 2006, ApJ, 653, 361

Le Bourlot, J., Le Petit, F., Pinto, C., Roueff, E., \& Roy, F. 2012, A\&A, 541,A76

Lopez, L. A., Krumholz, M. R., Bolatto, A. D., Prochaska, J. X., \& Ramirez-Ruiz, E. 2011, ApJ, 731, 91

Mengel, S., Lehnert, M. D., Thatte, N., \& Genzel, R. 2005, A\&\&A, 443, 41

Murray, N. 2011, ApJ, 729, 133

Murray, N., Quataert, E., \& Thompson, T. A. 2010, ApJ, 709, 191

Sakamoto, K., Ho, P. T. P., Iono, D., Keto, E. R., Mao, R.-Q., Matsushita, S., Peck, A. B., Wiedner, M. C., Wilner, D. J., \& Zhao, J.-H. 2006, ApJ, 636, 685

Snijders, L., van der Werf, P. P., Brandl, B. R., Mengel, S., Schaerer, D., \& Wang, Z. 2006, ApJ, 648, L25

Schweizer, F., Burns, C. R., Madore, B. F., Mager, V. A., Phillips, M. M., Freedman, W. L., Boldt, L., Contreras, C., Folatelli, G., González, S., Hamuy, M., Krzeminski, W., Morrell, N. I., Persson, S. E., Roth, M. R., \& Stritzinger, M. D. 2008, AJ, 136, 1482

Tan, J. C., Krumholz, M. R., \& McKee, C. F. 2006, ApJ, 641, L121

Weiss, A., Walter, F., Neininger, N., \& Klein, U. 1999, A\&A A, 345, L23

Whitmore, B. C., Brogan, C., Chandar, R., Evans, A., Hibbard, J., Johnson, K., Leroy, A., Privon, G., Remijan, A., \& Sheth, K. 2014, ApJ, 795, 156

Whitmore, B. C. \& Schweizer, F. 1995, AJ, 109, 960

Wilson, C., Scoville, N., Madden, S., \& Charmandaris, V. 2000, ApJ, 542, 120

Zezas, A., Fabbiano, G., Baldi, A., Schweizer, F., King, A. R., Ponman, T. J., \& Rots, A. H. 2006, ApJS, 166, 211

Zhu, M., Seaquist, E. R., \& Kuno, N. 2003, ApJ, 588, 243 